\title{
CLOSED CATEGORIES \\ GENERATED BY COMMUTATIVE MONADS
}

\author{
ANDERS KOCK \\ (Received 7 March 1969) \\ Communicated by G. B. Preston
}

\section{Introduction}

The notion of commutative monad was defined by the author in [4]. The content of the present paper may briefly be stated: The category of algebras for a commutative monad can in a canonical way be made into a closed category, the two adjoint functors connecting the category of algebras with the base category are in a canonical way closed functors, and the front- and end-adjunctions are closed transformations. (The terms 'Closed Category' etc. are from the paper [2] by Eilenberg and Kelly). In particular, the monad itself is a 'closed monad'; this fact was also proved in [4].

In section 1 and henceforth, $\mathscr{V}$ is a symmetric monoidal closed category; in this setting, the construction of the fundamental transformation $\lambda:(A \pitchfork B) T \rightarrow$ $A \pitchfork(B) T$ can take place ( $\pitchfork$ denoting the inner hom-functor of $\mathscr{V}$, and $T$ an arbitrary $\mathscr{V}$-endofunctor on $\mathscr{V}$ ). Some equations involving $\lambda$ are proved. These are used in sections 2 and 3 for the main construction.

We shall stick to the terminology and notation of [4], which is the same as the terminology of [2] except that the hom-object of $A$ and $B$ is denoted $A \pitchfork B$ instead of $(A B)$ or hom $\mathscr{V}(A, B)$.

\section{The transformation $\lambda$}

In this section, $\mathscr{V}$ denotes a symmetric monoidal closed category ([2], Chapter III), and $T$ a $\mathscr{V}$-endofunctor (strong endofunctor) on $\mathscr{V}$, just as in [4], Section 1 . There we constructed certain natural transformations $t^{\prime}$ and $t^{\prime \prime}$ associated with $T$. Essentially the same information will here be contained in the natural transformation:

$$
(A \pitchfork B) T \stackrel{\lambda_{A}, \boldsymbol{B}}{\longrightarrow} A \pitchfork(B) T,
$$

which we now shall construct by writing down a deduction scheme: 


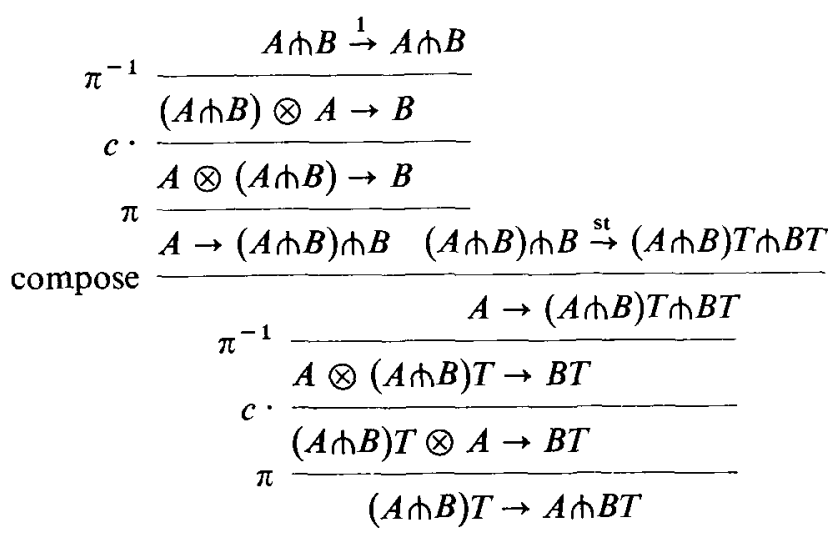

Here $c$. on the left of a line indicates 'composing on the left by the symmetry $c$ of the tensorproduct'; $\pi$ denotes the fundamental connection between $\pitchfork$ and $\otimes$ in a monoidal closed category; and st denotes the $\mathscr{V}$-functor structure of $T$. The meaning of (1.1) for stepwise construction of $\lambda$ should now be immediately understandable. The technique of deduction schemes was introduced by Lambek [5]. Let us denote the fourth arrow of the scheme, i.e. $A \rightarrow(A \pitchfork B) \pitchfork B$ by $g_{A}^{B}$. (It may be thought of as a generalization of the well-known 'embedding a vector space in its double dual').

We may of course replace the application of $\pi$ and $\pi^{-1}$ in this scheme by suitable compositions with the front- and end-adjunctions for the adjointness given by $\pi$ :

$$
-\otimes D+D \pitchfork-
$$

these adjunctions we denote as follows:

$$
C \stackrel{f C^{D}}{\longrightarrow} D \pitchfork(C \otimes D) \quad(D \pitchfork C) \otimes D \stackrel{e v C^{D}}{\longrightarrow} C ;
$$

in [2], they are denoted $u$ and $t$, respectively. They are natural in both variables (in the generalized sense of 'natural' from [1]).

Doing this, we may describe $\lambda_{A, B}$ as the composite

$$
\begin{aligned}
& (A \pitchfork B) T \stackrel{f^{A}(A \pitchfork B) T}{\longrightarrow} A \pitchfork[(A \pitchfork B) T \otimes A] \stackrel{1 \otimes c}{\longrightarrow} A \pitchfork[A \otimes(A \pitchfork B) T] \\
& \stackrel{1 \pitchfork\left[g_{A} B \otimes 1\right]}{\longrightarrow} A \pitchfork[((A \pitchfork B) \pitchfork B) \otimes(A \pitchfork B) T] \stackrel{1 \pitchfork[\mathrm{st} \otimes 1]}{\longrightarrow} \\
& \longrightarrow A \pitchfork[((A \pitchfork B) T \pitchfork B T) \otimes(A \pitchfork B) T] \stackrel{1 \pitchfork v_{B T^{(A \pitchfork B) T}}}{\longrightarrow} A \pitchfork B T .
\end{aligned}
$$

We want to assert that $\lambda_{A, B}$ is $\mathscr{V}$-natural in each variable separately. By [2], Theorem III.7.4, $f, c$, and $e v$ are $\mathscr{V}$-natural, and the $\mathscr{V}$-naturality of st follows from [2], Proposition III.7.6. Furthermore $g_{A}^{B}$ may be described as the composite

$$
\begin{aligned}
& \stackrel{\stackrel{f_{A} A \pitchfork B}{\longrightarrow}}{\longrightarrow}(A \pitchfork B) \pitchfork[A \otimes(A \pitchfork B)] \stackrel{1 \pitchfork c}{\longrightarrow}(A \pitchfork B) \pitchfork[(A \pitchfork B) \otimes A] \\
& \stackrel{1 \pitchfork_{e v_{B} A}}{\longrightarrow}(A \pitchfork B) \pitchfork B .
\end{aligned}
$$


So the whole composite (1.2) is made up as a composition of combinations of $\mathscr{V}$-natural transformations with $\mathscr{V}$-functors. The $\mathscr{V}$-naturality of (1.2) then follows from the Propositions III.5.1 and III.5.2 of [2], so that we have

THEOREM 1.1. The transformation $\lambda$ is $\mathscr{V}$-natural in each variable. In particular, it is natural in the ordinary sense.

To connect the results of this paper with those of [4], and in order to use the results from [4], we next establish the connection between $\lambda$ and the $t^{\prime}, t^{\prime \prime}$ of Section 1 in [4].

LEMMA 1.2. With $t^{\prime}, t^{\prime \prime}$ as in [4], the following diagrams commute

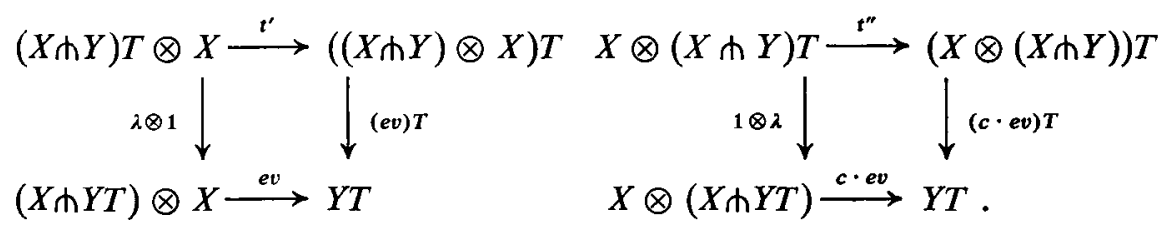

Proof. Since $t^{\prime}$ is defined in terms of $t^{\prime \prime}$ and $c$, it suffices to prove the second commutativity. We have by definition of $t^{\prime \prime}$ and naturality of st the commutative diagram

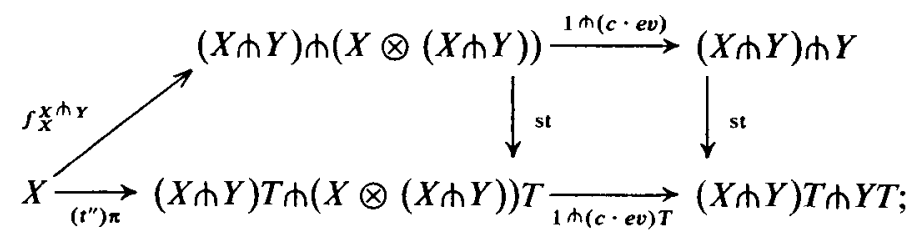

and by the formula (1.3), (1.4) expresses

$$
g_{X}^{Y} \cdot \mathrm{st}=\left(t^{\prime \prime}\right) \pi \cdot 1 \pitchfork(c \cdot e v) T .
$$

Applying $\pi^{-1}, c \cdot$, to the two sides of (1.5) gives the two legs of the right hand diagram of the Lemma, as is easily seen.

The following is a main lemma.

Lemma 1.3. The diagram

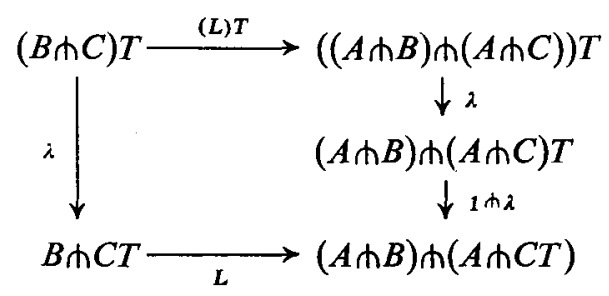

commutes. ( $L$ denoting 'composition' in $\mathscr{V}$, as in [2].)

Proof. We translate this equation to an equation between arrows from 
$(B \pitchfork C) T \otimes(A \pitchfork B) \otimes A$ to $C T$ by using the fundamental adjointness $\pi$. The translation of $\lambda \cdot L$ is the counterclockwise composite in

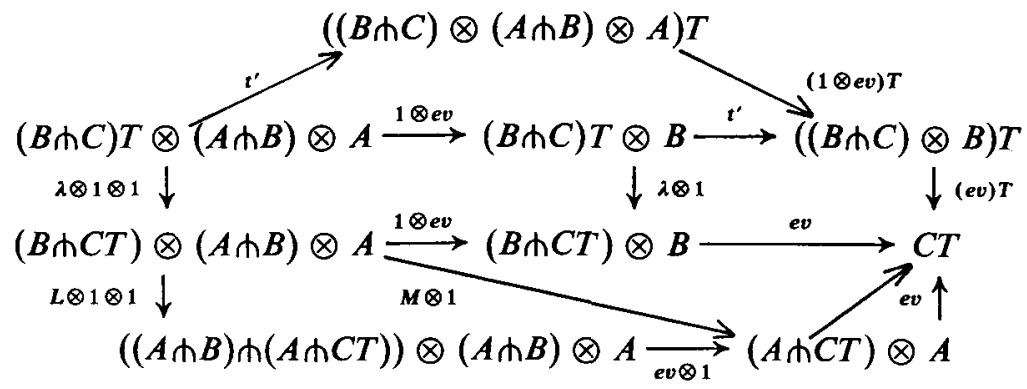

(The reader will easily fill in the associativity isomorphisms for $\otimes$. ) In this diagram, the top figure commutes by naturality of $t^{\prime}$; commutativity of the left square is obvious; for the right square, use Lemma 1.2. Finally, the bottom figure commutes by Lemma 1.3 of [4] (the triangle in the bottom figure is just the definition of $M$ ).

On the other hand, let us translate the composite $L T \cdot \lambda \cdot 1 \pitchfork \lambda$ of (1.6). It is the counterclockwise composite in

$(B \pitchfork C) T \otimes(A \pitchfork B) \otimes A \stackrel{t^{\prime} \otimes 1}{\longrightarrow}((B \pitchfork C) \otimes(A \pitchfork B)) T \otimes A \stackrel{t^{\prime}}{\longrightarrow}\left((B \pitchfork C) \otimes(A \pitchfork B) \otimes \prod A\right.$

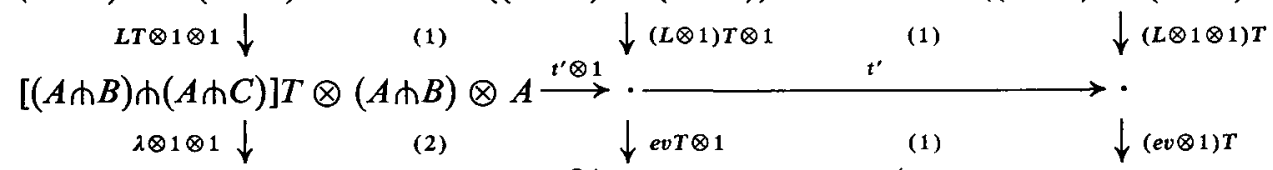

$[(A \pitchfork B) \pitchfork(A \pitchfork C) T] \otimes(A \pitchfork B) \otimes A \stackrel{e v \otimes 1}{\longrightarrow}(A \pitchfork C) T \otimes A \stackrel{t^{\prime}}{\longrightarrow}((A \pitchfork C) \otimes A) T$

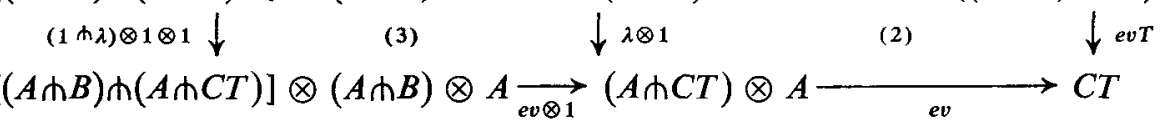

(The reader will here have to fill in some object names.) In this diagram, the top commutes by the fundamental Proposition 1.5 of [4]; the squares marked (1) commute by naturality of $t^{\prime}$. The squares marked (2) commute by Lemma 1.2. The square (3) commutes by naturality of $e v$. Finally, the right hand column may be rewritten $(1 \otimes e v) T \cdot(e v) T$, using exactly the same argument as for the bottom figure of (1.7). Doing this, the clockwise composite of (1.8) becomes the same as the clockwise composite of (1.7). This proves the lemma.

In analogy with Lemmas 1.1 and 1.2 of [4] we have the following two lemmas:

Lemma 1.4. Let $\alpha: T \Rightarrow S$ be a $\mathscr{V}$-natural transformation of $\mathscr{V}$-endofunctors on $\mathscr{V}$, i.e. $\alpha$ satisfies the axiom $\mathrm{VN}$ of [2]. Then the diagram 


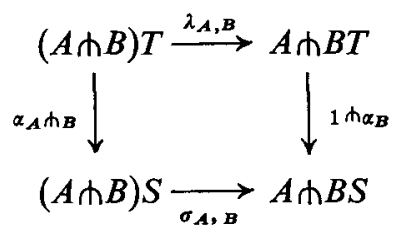

commutes, where $\sigma$ is associated to $S$ in the same way as $\lambda$ is associated to $T$.

Proof. Consider the description (1.2) of $\lambda$ (and the similar description of $\sigma$ ). Then one easily gets the desired commutativity, using ordinary naturality of $f$, ordinary and extraordinary naturality of $e v$, and $\mathscr{V}$-naturality of $\alpha$.

With notation as in this lemma we have

LEMMA 1.5. The transformation associated to $T \cdot S$ is the composite

$$
(A \pitchfork B) T S \stackrel{\lambda S}{\rightarrow}(A \pitchfork B T) S \stackrel{\sigma}{\rightarrow} A \pitchfork B T S .
$$

Proof. This is an easy consequence of the corresponding Lemma 1.2 in [4] for $t^{\prime}, s^{\prime}$, using the connection given in Lemma 1.2 here between $\lambda$ and $t^{\prime}$ (and between $\sigma$ and $s^{\prime}$ ).

As an immediate corollary of Lemmas 1.4 and 1.5 we have

LEMmA 1.6. If $\mu: T \cdot T \Rightarrow T$ and $\eta: 1_{\mathscr{r}} \Rightarrow$ Tare $\mathscr{V}$-natural, then $\mu \cdot \lambda=\lambda T \cdot \lambda \cdot$ $1 \pitchfork \mu$ and $\eta \cdot \lambda=1 \pitchfork \eta$.

LEMMA 1.7. With $i_{x}$ denoting that natural isomorphism $X \rightarrow I \pitchfork X$ which is part of the data of $\mathscr{V}$, the following diagram commutes

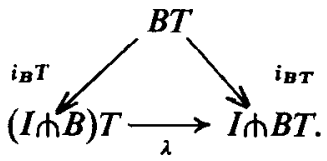

Proof. Consider the diagram

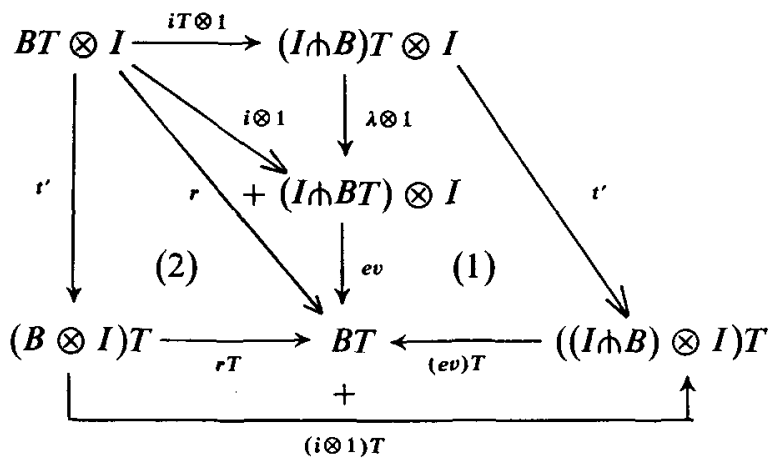

Here (1) commutes by Lemma 1.2, (2) by Lemma 1.8 in [4], the two diagrams 
marked + commute by Axiom MCC 4 of [2]. The outer diagram is just naturality of $t^{\prime}$. It follows that

$$
i_{B} T \otimes 1 \cdot \lambda \otimes 1 \cdot e v=i_{B T} \otimes 1 \cdot e v .
$$

From this the lemma follows.

\section{The closed category of algebras}

We now come to the main construction. We have to make an assumption on the symmetric monoidal closed category $\mathscr{V}$, namely that its underlying category $\mathscr{V}_{0}$ has equalizers. The 'underlying' functor $V$ from $\mathscr{V}_{0}$ to the category of sets preserves equalizers, since it is representable. We shall assume that we have for each pair of mappings $X \underset{g}{\stackrel{f}{\rightarrow}} Y$ a chosen equalizer $e_{f, g}$ which by $V$ is sent to the chosen equalizer of $f V, g V$ in the category of sets, (the chosen equalizers here being the appropriate inclusion mappings). This last assumption is a weak one.

We consider in the following a $\mathscr{V}$-monad $\mathbf{T}$ on $\mathscr{V}$, i.e. a triple $(T, \eta, \mu)$ where $T: \mathscr{V} \rightarrow \mathscr{V}$ is a $\mathscr{V}$-functor (as considered in the preceding section), and where $\eta$ and $\mu$ are $\mathscr{V}$-transformations

$$
\begin{array}{r}
1_{\mathscr{V}} \stackrel{\eta}{\rightarrow} T \\
T \cdot T \stackrel{\mu}{\rightarrow} T
\end{array}
$$

satisfying the usual equations

$$
\begin{aligned}
& \eta T \cdot \mu=T \eta \cdot \mu=1_{T} \\
& \mu T \cdot \mu=T \mu \cdot \mu .
\end{aligned}
$$

We shall furthermore assume that the monad is commutative in the following sense:

Definition 2.1. A $\mathscr{V}$-monad $\mathbf{T}$ on $\mathscr{V}$ is called commutative if the following diagram commutes

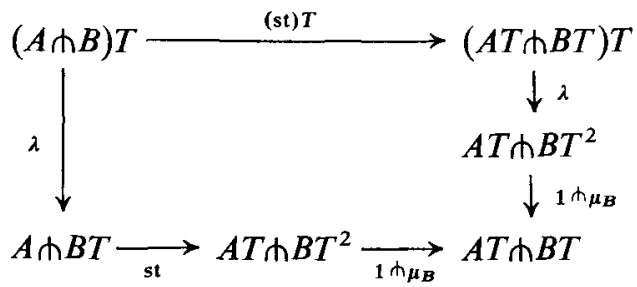

for all $A$ and $B$ in $\mathscr{V}_{0}$. The composite $(A \pitchfork B) T \rightarrow A T \pitchfork B T$ is denoted $\hat{T}_{A, B}$.

(This notion of commutativity can be shown to be the same as that of the previous paper [4].)

We shall now construct a closed category $\overline{\mathscr{V}}=\mathscr{V}^{\mathbf{T}}$ (generalizing the construction of [6]). By definition [2], this involves giving seven data; as a main 
rule, the data to be constructed have the same notation as the seven data for $\mathscr{V}$, except that we now put an upperbar.

(i) We must give the underlying category $\overline{\mathscr{V}}_{0}$; it is just taken to be $\mathscr{V}_{0}^{\mathbf{T}}$, i.e. the category constructed by Eilenberg and Moore in [3] for an ordinary monad $\mathbf{T}$ on the ordinary category $\mathscr{V}_{0}$. ('The category of algebras for $\mathbf{T}$ '). It has as its objects pairs $(A, a)$, where $A$ is an object of $\mathscr{V}_{0}$ and $a$ ('the structure') is a morphism

in $\mathscr{V}_{0}$ satisfying

$$
A T \stackrel{a}{\rightarrow} A
$$

$$
\begin{aligned}
& \eta_{A} \cdot a=1 \\
& a T \cdot a=\mu_{A} \cdot a .
\end{aligned}
$$

A morphism $(A, a) \rightarrow(B, b)$ in $\mathscr{V}_{0}=\mathscr{V}_{0}^{\mathbf{T}}$ is a morphism $f: A \rightarrow B$ in $\mathscr{V}_{0}$ such that $f T \cdot b=a \cdot f$. (Such an $f$ we shall call a homomorphism.) The category $\mathscr{V}_{0}^{\mathbf{T}}$ comes equipped with a functor $U: \mathscr{V}_{0}^{\mathbf{T}} \rightarrow \mathscr{V}_{0}$ given by $(A, a)-W \rightarrow A, f-W \rightarrow f$.

(ii) Let $\bar{V}$ denote the functor

$$
\overline{\mathscr{V}}_{0}=\mathscr{V}_{0}^{\mathbf{T}} \stackrel{U}{\rightarrow} \mathscr{V}_{0} \stackrel{\boldsymbol{V}}{\rightarrow} \mathscr{S}
$$

( $\mathscr{S}$ being the category of sets, $V$ being part (ii) of the data of $\mathscr{V}$ ).

For the remaining data (iii)-(vii), the existence of the postulated factorizations over the relevant equalizer $e$ is postponed till all the data is given.

(iii) We must give the internal hom functor of $\mathscr{V}$. Let $A=(A, a), B=(B, b)$ be objects of $\mathscr{V}_{0}^{\mathrm{T}}$. We construct an object $(A \bar{\pitchfork} B,\langle a, b\rangle)$ as follows. Let the following diagram be a chosen equalizer in $\mathscr{V}_{0}$

$$
A \bar{\pitchfork} B \stackrel{e_{A, B}}{\longrightarrow} A \pitchfork B \underset{\text { st }}{\longrightarrow} A T \pitchfork B T \underset{1 \pitchfork b}{\longrightarrow} A T \pitchfork B .
$$

Define the structure $\langle a, b\rangle$ by commutativity of the diagram

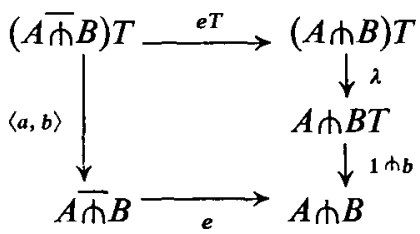

If $\beta: B \rightarrow B^{\prime}$ and $\alpha: A^{\prime} \rightarrow A$ are morphisms in $\mathscr{V}_{0}^{\mathbf{T}}$, we define a morphism $x \bar{\pitchfork} \beta$ by commutativity of the diagram

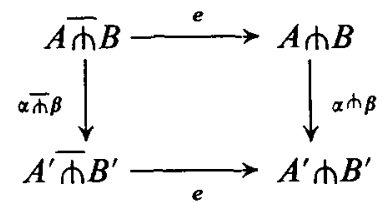

(iv) For base object $\bar{I}$ in $\mathscr{V}^{\mathrm{T}}$ we take $\bar{I}=\left(I T, \mu_{I}\right)$, where $I$ is the base object of $\mathscr{V}$. 
(v) We construct an (iso-)morphism $\bar{i}_{A}$ by commutativity of

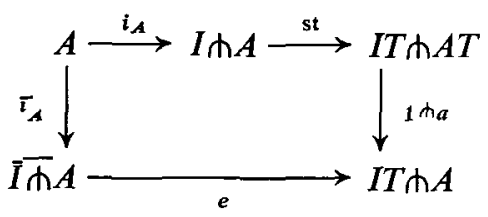

(vi) We construct a morphism $j_{A}$ by commutativity of

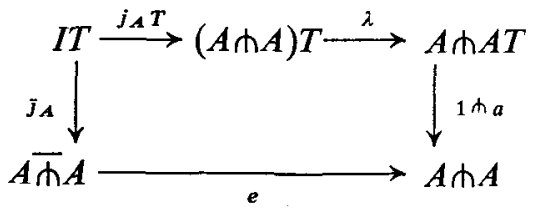

(vii) We construct a morphism $\bar{L}_{B C}^{A}$ by commutativity of

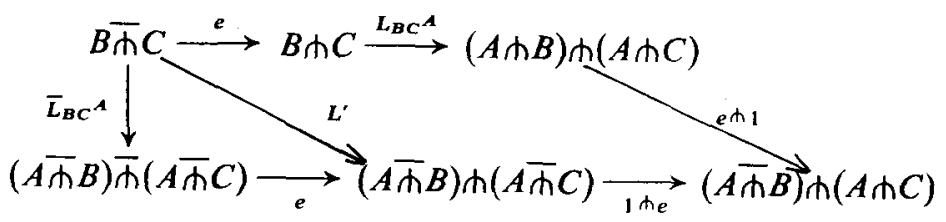

(in the bottom left corner, the middle $\bar{\pitchfork}$ indicates that $A \bar{\pitchfork} B$ and $A \bar{\pitchfork} C$ are considered together with their structures $\langle a, b\rangle$ and $\langle a, c\rangle$ respectively (where $C=(C, c))$.

This is the data for the closed category $\mathscr{V}^{\mathbf{T}}$. We now come to the verifications. First, we have to show that the defining expressions for the morphisms $\langle a, b\rangle$, $\alpha \bar{\pitchfork} \beta, i_{A}, \bar{j}_{A}, \bar{L}_{B C}^{A}$ express morphisms that actually factor through the relevant $c$; since $e$ is an equalizer, this means verifying an equation.

For $\langle a, b\rangle$, we must show that $e T \cdot \lambda \cdot 1 \pitchfork b$ equalizes $a \pitchfork 1$ and st $\cdot 1 \pitchfork b$. We shall write down a string of equations giving the result; the equality signs carry explanations: a $\lambda$ on top of the equality sign means: 'by naturality of $\lambda$. .' An 'st' means 'by naturality of st', and an $e$ means: by the equalizing property of $e$. A number, e.g. (2.3), refers to the equation of that number. $A *$ means 'Will be explained after the equation'. We then have

$$
\begin{aligned}
& e T \cdot \lambda \cdot 1 \pitchfork b \cdot a \pitchfork 1=e T \cdot \lambda \cdot a \pitchfork 1 \cdot 1 \pitchfork b \stackrel{\lambda}{=} e T \cdot(a \pitchfork 1) T \cdot \lambda \cdot 1 \pitchfork b \\
& \stackrel{e}{=} e T \cdot \mathrm{st} T \cdot(1 \pitchfork b) T \cdot \lambda \cdot 1 \pitchfork b \stackrel{\lambda}{=} e T \cdot \mathrm{st} T \cdot \lambda \cdot 1 \pitchfork b T \cdot 1 \pitchfork b \\
& \stackrel{(2.3)}{=} e T \cdot \mathrm{st} T \cdot \lambda \cdot 1 \pitchfork \mu \cdot 1 \pitchfork b \stackrel{*}{=} e T \cdot \lambda \cdot \mathrm{st} \cdot 1 \pitchfork \mu \cdot 1 \pitchfork b \\
& \stackrel{(2.3)}{=} e T \cdot \lambda \cdot \mathrm{st} \cdot 1 \pitchfork b T \cdot 1 \pitchfork b \stackrel{\text { st }}{=} e T \cdot \lambda \cdot 1 \pitchfork b \cdot \mathrm{st} \cdot 1 \pitchfork b .
\end{aligned}
$$

Here $*$ is the assumption (2.2) on commutativity of the monad. This proves the desired equation, and thus the existence of $\langle a, b\rangle$. Note that only $b$ was really used in defining the structure $\langle a, b\rangle$; this is an abstract version of the fact that to 
define the abelian group structure on the set of homomorphisms from an abelian group $A$ to an abelian group $B$, only the group structure of $B$ enters. The reader may exemplify the whole construction in this chapter by taking $\mathscr{V}$ to be the category of sets, $T$ to be the free-abelian-group monad (which is commutative). - We have to verify that $\langle a, b\rangle$ satisfies equations similar to (2.3). The first equation follows from

$$
\eta \cdot\langle a, b\rangle \cdot e \stackrel{D}{=} \eta \cdot e T \cdot \lambda \cdot 1 \pitchfork b \stackrel{\eta}{=} e \cdot \eta \cdot \lambda \cdot 1 \pitchfork b \stackrel{1.6}{=} e \cdot 1 \pitchfork \eta \cdot 1 \pitchfork b \stackrel{(2.3)}{=} e
$$

and the fact that $e$ is a monomorphism. We have here extended the conventions for the preceding string of equations to comprise: a ' $D$ ' means 'follows from the definitions', $\eta$ (or $\mu$ ) means by naturality of $\eta$ (or $\mu$ ), and a number 1.6 without bracket refers to the Lemma with that number. - The other equation of $(2.3)$ for $\langle a, b\rangle$ follows from

$$
\begin{aligned}
& \mu \cdot\langle a, b\rangle \cdot e \stackrel{D}{=} \mu \cdot e T \cdot \lambda \cdot 1 \pitchfork b \stackrel{\mu}{=} e T^{2} \cdot \mu \cdot \lambda \cdot 1 \pitchfork b \\
& \stackrel{1.6}{=} e T^{2} \cdot \lambda T \cdot \lambda \cdot 1 \pitchfork \mu \cdot 1 \pitchfork b \stackrel{(2.3)}{=} e T^{2} \cdot \lambda T \cdot \lambda \cdot 1 \pitchfork b T \cdot 1 \pitchfork b \\
& \stackrel{\lambda}{=} e T^{2} \cdot \lambda T \cdot(1 \pitchfork b) T \cdot \lambda \cdot 1 \pitchfork b \stackrel{D}{=}\langle a, b\rangle T \cdot e T \cdot \lambda \cdot 1 \pitchfork b \stackrel{D}{=}\langle a, b\rangle T \cdot\langle a, b\rangle \cdot e .
\end{aligned}
$$

The verification required for defining $\alpha \bar{\AA} \beta$ (where $\alpha$ and $\beta$ are homomorphisms) is very easy and left to the reader. But we also have to verify that $\alpha \bar{\pitchfork} \beta$ is a homomorphism. This follows from

$$
\begin{aligned}
& \langle a, b\rangle \cdot \alpha \bar{\pitchfork} \beta \cdot e \stackrel{D}{=}\langle a, b\rangle \cdot e \cdot \alpha \pitchfork \beta \stackrel{D}{=} e T \cdot \lambda \cdot 1 \pitchfork b \cdot \alpha \pitchfork \beta \\
& =e T \cdot \lambda \cdot \alpha \pitchfork 1 \cdot 1 \pitchfork b \cdot 1 \pitchfork \beta \stackrel{*}{=} e T \cdot \lambda \cdot \alpha \pitchfork 1 \cdot 1 \pitchfork \beta T \cdot 1 \pitchfork b^{\prime} \\
& \stackrel{\lambda}{=} e T \cdot(\alpha \pitchfork 1) T \cdot \lambda \cdot 1 \pitchfork \beta T \cdot 1 \pitchfork b^{\prime} \stackrel{\lambda}{=} e T \cdot(\alpha \pitchfork 1) T \cdot(1 \pitchfork \beta) T \cdot \lambda \cdot 1 \pitchfork b^{\prime} \\
& \stackrel{D}{=}(\alpha \bar{\pitchfork} \beta) T \cdot e T \cdot \lambda \cdot 1 \pitchfork b^{\prime} \stackrel{D}{=}(\alpha \pitchfork \pitchfork) T \cdot\left\langle a^{\prime}, b^{\prime}\right\rangle \cdot e,
\end{aligned}
$$

the equality $\operatorname{sign} *$ because $\beta$ is a homomorphism.

Next we turn to the definition of $\bar{i}_{\boldsymbol{A}}$. We have to prove that $i_{\boldsymbol{A}} \cdot \mathrm{st} \cdot 1$ ha equalizes $\mu_{I} \pitchfork 1$ and st 1 i $\pitchfork a$. We have

$$
\begin{aligned}
& i_{A} \cdot \mathrm{st} \cdot 1 \pitchfork a \cdot \mu \pitchfork 1=i_{A} \cdot \mathrm{st} \cdot \mu \pitchfork 1 \cdot 1 \pitchfork a \stackrel{*}{=} i_{A} \cdot \mathrm{st} \cdot \mathrm{st} \cdot 1 \pitchfork \mu \cdot 1 \pitchfork a \\
& \stackrel{(2.3)}{=} i_{A} \cdot \mathrm{st} \cdot \mathrm{st} \cdot 1 \pitchfork a T \cdot 1 \pitchfork a \stackrel{\mathrm{st}}{=} i_{A} \cdot \mathrm{st} \cdot 1 \pitchfork a \cdot \mathrm{st} \cdot 1 \pitchfork a,
\end{aligned}
$$

* being the $\mathscr{V}$-naturality of $\mu$.

We want to know that $\bar{i}_{\boldsymbol{A}}$ is a homomorphism and is invertible. We claim that

$$
k: \bar{I} \bar{\pitchfork} A \stackrel{e}{\longrightarrow} I T \pitchfork A \stackrel{\eta_{I} \pitchfork 1}{\longrightarrow} I \pitchfork A \stackrel{i^{-1}}{\longrightarrow} A
$$

is the inverse of $i_{A}$. For 


$$
\begin{aligned}
& i \cdot k \stackrel{D}{=} i \cdot e \cdot \eta \pitchfork 1 \cdot i^{-1} \stackrel{D}{=} i \cdot \mathrm{st} \cdot 1 \pitchfork a \cdot \eta \pitchfork 1 \cdot i^{-1} \\
& =i \cdot \mathrm{st} \cdot \eta \pitchfork 1 \cdot 1 \pitchfork a \cdot i^{-1} \stackrel{i}{=} i \cdot \mathrm{st} \cdot \eta \pitchfork 1 \cdot i^{-1} \cdot a \\
& \stackrel{*}{=} i \cdot 1 \pitchfork \eta \cdot i^{-1} \cdot a \stackrel{i}{=} \eta \cdot a \stackrel{(2.3)}{=} 1
\end{aligned}
$$

* being the $\mathscr{V}$-naturality of $\eta$. To prove $k \cdot \bar{i}=1$, consider

$$
\begin{aligned}
& k \cdot i \cdot e \stackrel{D}{=} k \cdot i \cdot \mathrm{st} \cdot 1 \pitchfork a \stackrel{D}{=} e \cdot \eta \pitchfork 1 \cdot \mathrm{st} \cdot 1 \pitchfork a \stackrel{\text { st }}{=} e \cdot \mathrm{st} \cdot \eta T \pitchfork 1 \cdot 1 \pitchfork a \\
& =e \cdot \mathrm{st} \cdot 1 \pitchfork a \cdot \eta T \pitchfork 1 \stackrel{e}{=} e \cdot \mu \pitchfork 1 \cdot \eta T \pitchfork 1 \stackrel{(2.1)}{=} e .
\end{aligned}
$$

From this $k \cdot i=1$ follows. We shall prove now that $k$ is a homomorphism (then automatically, $i$ will be so). We have

$$
\begin{aligned}
& \langle\mu, a\rangle \cdot k \stackrel{D}{=}\langle\mu, a\rangle \cdot e \cdot \eta \pitchfork 1 \cdot i^{-1} \stackrel{D}{=} e T \cdot \lambda \cdot 1 \pitchfork a \cdot \eta \pitchfork 1 \cdot i^{-1} \\
& =e T \cdot \lambda \cdot \eta \pitchfork 1 \cdot 1 \pitchfork a \cdot i^{-1} \stackrel{\lambda}{=} e T \cdot(\eta \pitchfork 1) T \cdot \lambda \cdot 1 \pitchfork a \cdot i^{-1} \\
& \stackrel{i}{=} e T \cdot(\eta \pitchfork 1) T \cdot \lambda \cdot i^{-1} \cdot a \stackrel{1.7}{=} e T \cdot(\eta \pitchfork 1) T \cdot\left(i^{-1}\right) T \cdot a \stackrel{D}{=} k T \cdot a .
\end{aligned}
$$

Clearly $k$ (and therefore $i$ ) is natural in $A$ (with respect to homomorphisms).

We next turn to $j$; the existence of a $j$ making (2.4) commutative follows from the equations

$$
\begin{aligned}
& j_{A} T \cdot \lambda \cdot 1 \pitchfork a \cdot \mathrm{st} \cdot 1 \mathrm{ha} \stackrel{\mathrm{st}}{=} j_{A} T \cdot \lambda \cdot \mathrm{st} \cdot 1 \mathrm{~h} a T \cdot 1 \pitchfork a \\
& \stackrel{(2.3)}{=} j_{A} T \cdot \lambda \cdot \mathrm{st} \cdot 1 \pitchfork \mu \cdot 1 \pitchfork a \stackrel{*}{=} j_{A} T \cdot(\mathrm{st}) T \cdot \lambda \cdot 1 \pitchfork \mu \cdot 1 \pitchfork a \\
& \stackrel{(2.3)}{=} j_{A} T \cdot \mathrm{st} T \cdot \lambda \cdot 1 \pitchfork a T \cdot 1 \pitchfork a \stackrel{\lambda}{=} j_{A} T \cdot \mathrm{st} T \cdot(1 \pitchfork a) T \cdot \lambda \cdot 1 \pitchfork a \\
& \stackrel{v F 1}{=}\left(j_{A T} \cdot 1 \pitchfork a\right) T \cdot \lambda \cdot 1 \pitchfork a \stackrel{j}{=}\left(j_{A} \cdot a \pitchfork 1\right) T \cdot \lambda \cdot 1 \pitchfork a \stackrel{\lambda}{=} j_{A} T \cdot \lambda \cdot a \pitchfork 1 \cdot 1 \pitchfork a \\
& =j_{A} T \cdot \lambda \cdot 1 \pitchfork a \cdot a \pitchfork 1,
\end{aligned}
$$

where the equality $\operatorname{sign} *$ is by commutativity of the monad, and 'VF 1' means 'VF 1 for the functor $T$ '. The fact that $j$ is a homomorphism follows from the equations

$$
\begin{aligned}
& \mu_{I} \cdot j \cdot e \stackrel{D}{=} \mu \cdot j T \cdot \lambda \cdot 1 \pitchfork a \stackrel{\mu}{=} j T^{2} \cdot \mu \cdot \lambda \cdot 1 \pitchfork a \\
& \stackrel{\underline{1.6}}{=} j T^{2} \cdot \lambda T \cdot \lambda \cdot 1 \pitchfork \mu \cdot 1 \pitchfork a \stackrel{(2.3)}{=} j T^{2} \cdot \lambda T \cdot \lambda \cdot 1 \pitchfork a T \cdot 1 \pitchfork a \\
& \stackrel{\lambda}{=} j T^{2} \cdot \lambda T \cdot(1 \pitchfork a) T \cdot \lambda \cdot 1 \pitchfork a \stackrel{D}{=} j T \cdot e T \cdot \lambda \cdot 1 \pitchfork a \stackrel{D}{=} j T \cdot\langle a, a\rangle \cdot e .
\end{aligned}
$$

To check that $j$ is natural with respect to homomorphisms is straightforward from the naturality of $j$ and omitted.

Finally, we turn to the existence of a diagram (2.5). We first prove the existence of the arrow $L^{\prime}$ in that diagram; for this purpose, we use the fact that 1 he in that diagram is an equalizer of $1 \pitchfork(s t \cdot 1 \pitchfork c)$ and $1 \pitchfork(a \pitchfork 1)\left(X \pitchfork-: \mathscr{V}_{0} \rightarrow \mathscr{V}_{0}\right.$ preserves equalizers since it has an adjoint $-\otimes X)$. We have 


$$
\begin{aligned}
& e \cdot L^{A} \cdot e \pitchfork 1 \cdot 1 \pitchfork \mathrm{st} \cdot 1 \pitchfork(1 \pitchfork c)=e \cdot L^{A} \cdot 1 \pitchfork \mathrm{st} \cdot e \pitchfork 1 \cdot 1 \pitchfork(1 \pitchfork c) \\
& \stackrel{\underline{V F 2}}{=} e \cdot \mathrm{st} \cdot L^{A T} \cdot \mathrm{sth} 1 \cdot e \pitchfork 1 \cdot 1 \pitchfork(1 \pitchfork c)=e \cdot \mathrm{st} \cdot L^{A T} \cdot 1 \pitchfork(1 \pitchfork c) \cdot \mathrm{sth} 1 \cdot e \pitchfork 1 \\
& \stackrel{L}{=} e \cdot \mathrm{st} \cdot 1 \pitchfork c \cdot L^{A T} \cdot \mathrm{sth} 1 \cdot e \pitchfork 1 \stackrel{e}{=} e \cdot b \pitchfork 1 \cdot L^{A T} \cdot \mathrm{sth1} \cdot e \pitchfork 1 \\
& \stackrel{L}{=} e \cdot L^{A T} \cdot(1 \pitchfork b) \pitchfork 1 \cdot \mathrm{sth} 1 \cdot e \pitchfork 1 \stackrel{e \pitchfork 1}{=} e \cdot L^{A T} \cdot(a \pitchfork 1) \pitchfork 1 \cdot e \pitchfork 1 \\
& \stackrel{L}{=} e \cdot L^{A} \cdot 1 \pitchfork(a \pitchfork 1) \cdot e \pitchfork 1=e \cdot L^{A} \cdot e \pitchfork 1 \cdot 1 \pitchfork(a \pitchfork 1) .
\end{aligned}
$$

This gives the desired $L^{\prime}$. (Note that commutativity of the monad does not enter at this stage; in fact, for any $\mathscr{V}$-monad $\mathbf{T}$ on a $\mathscr{V}$-category $\mathscr{A}$, the $L^{\prime}$ constructed as here can be used to make the category of algebras over the monad into a $\mathscr{V}$ category with hom $\mathscr{A}^{\mathbf{T}}(A, B)=A \bar{\pitchfork} B \in \mathscr{V}$.) - We now have to prove that $L^{\prime}$ factors through $e$, i.e. that $L^{\prime}$ equalizes $\langle a, b\rangle \pitchfork 1$ and st $1 \pitchfork\langle a, c\rangle$. Since 1 he is a monomorphism, it suffices to prove

$$
L^{\prime} \cdot \mathrm{st} \cdot 1 \pitchfork\langle a, c\rangle \cdot 1 \pitchfork e=L^{\prime} \cdot\langle a, b\rangle \pitchfork 1 \cdot 1 \pitchfork e .
$$

The left hand side here is by definition

$$
\begin{aligned}
& L^{\prime} \cdot \mathrm{st} \cdot 1 \pitchfork e T \cdot 1 \pitchfork \lambda \cdot 1 \pitchfork(1 \pitchfork c) \stackrel{\mathrm{st}}{=} L^{\prime} \cdot 1 \pitchfork e \cdot \mathrm{st} \cdot 1 \pitchfork \lambda \cdot 1 \pitchfork(1 \pitchfork c) \\
& \stackrel{D}{=} e \cdot L \cdot e \pitchfork 1 \cdot \mathrm{st} \cdot 1 \pitchfork \lambda \cdot 1 \pitchfork(1 \pitchfork c) \\
& \stackrel{\mathrm{st}}{=} e \cdot L \cdot \mathrm{st} \cdot e T \pitchfork 1 \cdot 1 \pitchfork \lambda \cdot 1 \pitchfork(1 \pitchfork c) \\
& =e \cdot L \cdot \mathrm{st} \cdot 1 \pitchfork \lambda \cdot 1 \pitchfork(1 \pitchfork c) \cdot e T \pitchfork 1 \\
& \stackrel{*}{=} e \cdot \mathrm{st} \cdot L \cdot \lambda \pitchfork 1 \cdot 1 \pitchfork(1 \pitchfork c) \cdot e T \pitchfork 1 \\
& =e \cdot \mathrm{st} \cdot L \cdot 1 \pitchfork(1 \pitchfork c) \cdot \lambda \pitchfork 1 \cdot e T \pitchfork 1 \\
& \stackrel{L}{=} e \cdot \mathrm{st} \cdot 1 \pitchfork c \cdot L \cdot \lambda \pitchfork 1 \cdot e T \pitchfork 1 \\
& \stackrel{e}{=} e \cdot b \pitchfork 1 \cdot L \cdot \lambda \pitchfork 1 \cdot e T \pitchfork 1 \\
& \stackrel{L}{=} e \cdot L \cdot(1 \pitchfork b) \pitchfork 1 \cdot \lambda \pitchfork 1 \cdot e T \pitchfork 1 \\
& \stackrel{D}{=} e \cdot L \cdot e \pitchfork 1 \cdot\langle a, b\rangle \pitchfork 1 \\
& \stackrel{D}{=} L^{\prime} \cdot 1 \pitchfork e \cdot\langle a, b\rangle \pitchfork 1=L^{\prime} \cdot\langle a, b\rangle \pitchfork 1 \cdot 1 \pitchfork e
\end{aligned}
$$

which is the right hand side of (2.6). The equality sign marked $*$ is by $\mathscr{V}$-naturality of $\lambda$ (Theorem 1.1). This proves the existence of the morphism $\bar{L}_{B C}^{A}$. We have to see that it is a homomorphism. Since $e \cdot 1$ he is a monomorphism, it suffices to prove

$$
\bar{L} T \cdot\langle\langle a, b\rangle,\langle a, c\rangle\rangle \cdot e \cdot 1 \hbar e=\langle b, c\rangle \cdot \bar{L} \cdot e \cdot 1 \text { he. }
$$

We have for the left hand side

$$
\begin{aligned}
& \bar{L} T \cdot\langle\langle a, b\rangle,\langle a, c\rangle\rangle \cdot e \cdot 1 \pitchfork e \stackrel{D}{=} L T \cdot e T \cdot \lambda \cdot 1 \pitchfork\langle a, c\rangle \cdot 1 \pitchfork e \\
& \stackrel{D}{=} L T \cdot e T \cdot \lambda \cdot 1 \pitchfork e T \cdot 1 \pitchfork \lambda \cdot 1 \pitchfork(1 \pitchfork c) \stackrel{\lambda}{=} L T \cdot e T \cdot(1 \pitchfork e) T \cdot \lambda \cdot 1 \pitchfork \lambda \cdot 1 \pitchfork(1 \pitchfork c) \\
& \stackrel{D}{=} e T \cdot L T \cdot(e \pitchfork 1) T \cdot \lambda \cdot 1 \pitchfork \lambda \cdot 1 \pitchfork(1 \pitchfork c)
\end{aligned}
$$




$$
\begin{aligned}
& \stackrel{\lambda}{=} e T \cdot L T \cdot \lambda \cdot e \pitchfork 1 \cdot 1 \pitchfork \lambda \cdot 1 \pitchfork(1 \pitchfork c)=e T \cdot L T \cdot \lambda \cdot 1 \pitchfork \lambda \cdot e \pitchfork 1 \cdot 1 \pitchfork(1 \pitchfork c) \\
& =e T \cdot L T \cdot \lambda \cdot 1 \pitchfork \lambda \cdot 1 \pitchfork(1 \pitchfork c) \cdot e \pitchfork 1 \stackrel{1.3}{=} e T \cdot \lambda \cdot L \cdot 1 \pitchfork(1 \pitchfork c) \cdot e \pitchfork 1 \\
& \stackrel{L}{=} e T \cdot \lambda \cdot 1 \pitchfork c \cdot L \cdot e \pitchfork 1 \stackrel{D}{=}\langle b, c\rangle \cdot e \cdot L \cdot e \pitchfork 1 \stackrel{D}{=}\langle b, c\rangle \cdot L \cdot e \cdot 1 \pitchfork e
\end{aligned}
$$

which is just the right hand side of (2.7).

The proof that $\bar{L}$ is natural with respect to homomorphisms follows from the fact that $e$ is a monomorphism and natural with respect to homomorphisms, and from $L$ 's naturality. We omit the details.

TheOREM 2.2. The data (i)-(vii) defined above for $\mathscr{V}^{\mathbf{T}}$ makes $\mathscr{V}^{\mathbf{T}}$ into a closed category.

Proof. It just remains to verify the axioms $\mathrm{CC} 0-\mathrm{CC} 5$ of [2]. The axioms CC1-CC4 say that certain diagrams in $\mathscr{V}^{\mathbf{T}}$ commute. Since there is an 'underlying' functor $U: \mathscr{V}_{0}^{\mathbf{T}} \rightarrow \mathscr{V}_{0}$ which is faithful, it suffices to check the commutativities in $\mathscr{V}_{0}$.

Axiom $\mathrm{CC} 0$ is concerned with a diagram of functors: The internal hom-functor followed by $V$ should give the ordinary hom-functor. It is here easily seen to be true, since $V: \mathscr{V}_{0} \rightarrow$ sets is assumed to preserve equalizers exactly.

Axiom $\mathrm{CC} 1$ says that

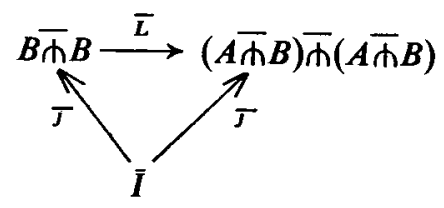

should commute. It follows if we can prove

$$
\bar{j}_{B} \cdot \bar{L}^{A} \cdot e \cdot 1 \text { he }=\bar{j}_{A} \pitchfork_{B} \cdot e \cdot 1 \text { he. }
$$

The left hand side here is

$$
\begin{aligned}
& j_{B} \cdot \bar{L} \cdot e \cdot 1 \pitchfork e \stackrel{D}{=} j_{B} \cdot e \cdot L \cdot e \pitchfork 1 \stackrel{D}{=} j_{B} T \cdot \lambda \cdot 1 \pitchfork b \cdot L \cdot e \pitchfork 1 \\
& \stackrel{L}{=} j_{B} T \cdot \lambda \cdot L \cdot 1 \pitchfork(1 \pitchfork b) \cdot e \pitchfork 1 \stackrel{1.3}{=} j_{B} T \cdot L T \cdot \lambda \cdot 1 \pitchfork \lambda \cdot 1 \pitchfork(1 \pitchfork b) \cdot e \pitchfork 1 \\
& \stackrel{C C 1}{=}\left(j_{A \pitchfork B}\right) T \cdot \lambda \cdot 1 \pitchfork \lambda \cdot 1 \pitchfork(1 \pitchfork b) \cdot e \pitchfork 1=\left(j_{A \pitchfork B}\right) T \cdot \lambda \cdot e \pitchfork 1 \cdot 1 \pitchfork \lambda \cdot 1 \pitchfork(1 \pitchfork b) \\
& \stackrel{\lambda}{=}\left(j_{A \pitchfork B}\right) T \cdot(e \pitchfork 1) T \cdot \lambda \cdot 1 \pitchfork \lambda \cdot 1 \pitchfork(1 \pitchfork b) \\
& \stackrel{\underline{J}}{=}\left(j_{A \pitchfork B}\right) T \cdot(1 \pitchfork e) T \cdot \lambda \cdot 1 \pitchfork \lambda \cdot 1 \pitchfork(1 \pitchfork b) \\
& \stackrel{\lambda}{=}\left(j_{A \pitchfork B}\right) T \cdot \lambda \cdot 1 \pitchfork e T \cdot 1 \pitchfork \lambda \cdot 1 \pitchfork(1 \pitchfork b) \stackrel{D}{=}\left(j_{A \pitchfork B}\right) T \cdot \lambda \cdot 1 \pitchfork\langle a, b\rangle \cdot 1 \pitchfork e \\
& \stackrel{D}{=} \bar{j}_{A \bar{A} B} \cdot e \cdot 1 \pitchfork e
\end{aligned}
$$

which is just the right hand side of the desired equation. The equality sign marked $\mathrm{CCl}$ is by Axiom $\mathrm{CCl}$ for $\mathscr{V}$. 
Axiom $\mathrm{CC} 2$ says that

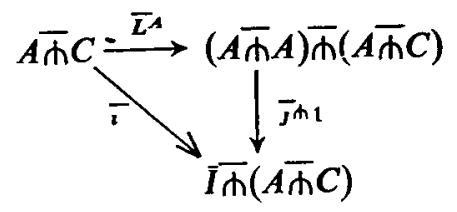

should commute. This follows if we can prove

$$
\bar{L}^{A} \cdot \overline{j \pitchfork} 1 \cdot e \cdot 1 \text { he }=\bar{i} \cdot e \cdot 1 \text { he. }
$$

The left hand side is

$$
\begin{aligned}
& L^{A} \cdot j \pitchfork 1 \cdot e \cdot 1 \pitchfork e \stackrel{D}{=} L \cdot e \cdot j \pitchfork 1 \cdot 1 \pitchfork e \\
& =L \cdot e \cdot 1 \pitchfork e \cdot j \pitchfork 1 \stackrel{D}{=} e \cdot L \cdot e \pitchfork 1 \cdot j \pitchfork 1 \stackrel{D}{=} e \cdot L \cdot(1 \pitchfork a) \pitchfork 1 \cdot \lambda \pitchfork 1 \cdot j T \pitchfork 1 \\
& \stackrel{L}{=} e \cdot a \pitchfork 1 \cdot L \cdot \lambda \pitchfork 1 \cdot j T \pitchfork 1 \stackrel{e}{=} e \cdot \mathrm{st} \cdot 1 \pitchfork c \cdot L \cdot \lambda \pitchfork 1 \cdot j T \pitchfork 1 \\
& \stackrel{L}{=} e \cdot \mathrm{st} \cdot L \cdot 1 \pitchfork(1 \pitchfork c) \cdot \lambda \pitchfork 1 \cdot j T \pitchfork 1=e \cdot \mathrm{st} \cdot L \cdot \lambda \pitchfork 1 \cdot j T \pitchfork 1 \cdot 1 \pitchfork(1 \pitchfork c) \\
& \stackrel{*}{=} e \cdot L \cdot \mathrm{st} \cdot 1 \pitchfork \lambda \cdot j T \pitchfork 1 \cdot 1 \pitchfork(1 \pitchfork c)=e \cdot L \cdot \mathrm{st} \cdot j T \pitchfork 1 \cdot 1 \pitchfork \lambda \cdot 1 \pitchfork(1 \pitchfork c) \\
& \stackrel{\mathrm{st}}{=} e \cdot L \cdot j \pitchfork 1 \cdot \mathrm{st} \cdot 1 \pitchfork \lambda \cdot 1 \pitchfork(1 \pitchfork c) \stackrel{c c 2}{=} e \cdot i \cdot \mathrm{st} \cdot 1 \pitchfork \lambda \cdot 1 \pitchfork(1 \pitchfork c) \\
& \stackrel{i}{=} i \cdot 1 \text { he } \cdot \mathrm{st} \cdot 1 \pitchfork \lambda \cdot 1 \pitchfork(1 \pitchfork c) \stackrel{\mathrm{st}}{=} i \cdot \mathrm{st} \cdot 1 \pitchfork e T \cdot 1 \pitchfork \lambda \cdot 1 \pitchfork(1 \pitchfork c) \\
& \stackrel{D}{=} i \cdot \mathrm{st} \cdot 1 \pitchfork\langle a, c\rangle \cdot 1 \pitchfork e \stackrel{D}{=} i \cdot e \cdot 1 \text { he }
\end{aligned}
$$

which is just the right hand side of the desired equation; the equality sign marked $*$ is by $\mathscr{V}$-naturality of $\lambda$ in the second variable (Theorem 1.1); the equality sign marked $\mathrm{CC} 2$ is by Axiom $\mathrm{CC} 2$ for $\mathscr{V}$.

Axiom CC3 expresses associativity of the 'composition' $L$ :

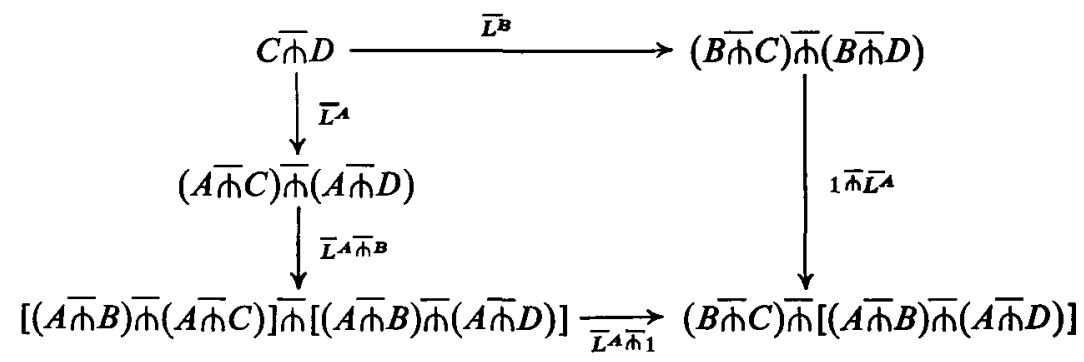

The commutativity of this diagram follows if we can prove

$$
\bar{L}^{B} \cdot 1 \bar{\hbar} \bar{L}^{A} \cdot e \cdot 1 \text { he } \cdot 1 \pitchfork(1 \pitchfork e)=\bar{L}^{A} \cdot \bar{L}^{A^{\AA_{B}}} \cdot \bar{L}^{A} \bar{\hbar} 1 \cdot e \cdot 1 \text { he } \cdot 1 \pitchfork(1 \text { he }) .
$$

The left hand side is

$$
\begin{aligned}
& L \cdot 1 \pitchfork \bar{L} \cdot e \cdot 1 \pitchfork e \cdot 1 \pitchfork(1 \pitchfork e) \stackrel{D}{=} \bar{L} \cdot e \cdot 1 \pitchfork \bar{L} \cdot 1 \pitchfork e \cdot 1 \pitchfork(1 \pitchfork e) \\
& \stackrel{D}{=} \bar{L} \cdot e \cdot 1 \pitchfork e \cdot 1 \pitchfork L \cdot 1 \pitchfork(e \pitchfork 1) \stackrel{D}{=} e \cdot L \cdot e \pitchfork 1 \cdot 1 \pitchfork L \cdot 1 \pitchfork(e \pitchfork 1)
\end{aligned}
$$


$=e \cdot L \cdot 1 \pitchfork L \cdot 1 \pitchfork(e \pitchfork 1) \cdot e \pitchfork 1 \stackrel{c c 3}{=} e \cdot L \cdot L \cdot L \pitchfork 1 \cdot 1 \pitchfork(e \pitchfork 1) \cdot e \pitchfork 1$

$=e \cdot L \cdot L \cdot 1 \pitchfork(e \pitchfork 1) \cdot L \pitchfork 1 \cdot e \pitchfork 1 \stackrel{L}{=} e \cdot L \cdot L \cdot(e \pitchfork 1) \pitchfork 1 \cdot L \pitchfork 1 \cdot e \pitchfork 1$

$\stackrel{D}{=} e \cdot L \cdot L \cdot(1 \pitchfork e) \pitchfork 1 \cdot e \pitchfork 1 \cdot L \pitchfork 1 \stackrel{L}{=} e \cdot L \cdot e \pitchfork 1 \cdot L \cdot e \pitchfork 1 \cdot \bar{L} \pitchfork 1$

$\stackrel{D}{=} \bar{L} \cdot e \cdot 1 \pitchfork e \cdot L \cdot e \pitchfork 1 \cdot \bar{L} \pitchfork 1 \stackrel{L}{=} \bar{L} \cdot e \cdot L \cdot 1 \pitchfork(1 \pitchfork e) \cdot e \pitchfork 1 \cdot \bar{L} \pitchfork 1$

$=L \cdot e \cdot L \cdot e \pitchfork 1 \cdot \bar{L} \pitchfork 1 \cdot 1 \pitchfork(1 \pitchfork e) \stackrel{D}{=} \bar{L} \cdot \bar{L} \cdot e \cdot 1 \pitchfork e \cdot \bar{L} \pitchfork 1 \cdot 1 \pitchfork(1 \pitchfork e)$

$=\bar{L} \cdot \bar{L} \cdot e \cdot L \pitchfork 1 \cdot 1 \pitchfork e \cdot 1 \pitchfork(1 \pitchfork e) \stackrel{D}{=} \bar{L} \cdot \bar{L} \cdot \bar{L} \bar{\hbar} 1 \cdot e \cdot 1 \pitchfork e \cdot 1 \pitchfork(1 \pitchfork e)$

which is just the right hand side of the desired equation. The equality sign marked $\mathrm{CC} 3$ is by Axiom $\mathrm{CC} 3$ for $\mathscr{V}$.

Axiom CC4 says that the following diagram should commute:

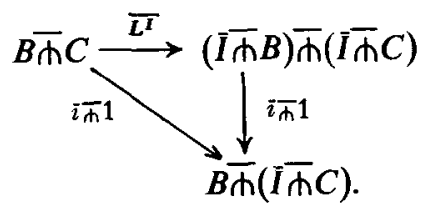

We have

$$
\begin{aligned}
& \bar{L} \bar{I} \cdot \bar{i} \pitchfork 1 \cdot e \cdot 1 \pitchfork e \stackrel{D}{=} \bar{L} \cdot e \cdot i \pitchfork 1 \cdot 1 \text { he }=\bar{L} \cdot e \cdot 1 \pitchfork e \cdot i \pitchfork 1 \\
& \stackrel{D}{=} e \cdot L \cdot e \pitchfork 1 \cdot i \pitchfork 1 \stackrel{D}{=} e \cdot L \cdot(1 \pitchfork b) \pitchfork 1 \cdot \operatorname{sth} 1 \cdot i \pitchfork 1 \\
& \stackrel{L}{=} e \cdot b \pitchfork 1 \cdot L \cdot \operatorname{sth} 1 \cdot I \pitchfork 1 \stackrel{e}{=} e \cdot \mathrm{st} \cdot 1 \pitchfork c \cdot L \cdot \operatorname{sth} 1 \cdot i \pitchfork 1 \\
& \stackrel{L}{=} e \cdot \mathrm{st} \cdot L \cdot 1 \pitchfork(1 \pitchfork c) \cdot \operatorname{sth} 1 \cdot i \pitchfork 1=e \cdot \mathrm{st} \cdot L \cdot \operatorname{sth} 1 \cdot i \pitchfork 1 \cdot 1 \pitchfork(1 \pitchfork c) \\
& \underline{\underline{V F 2}} e \cdot L \cdot 1 \pitchfork s t \cdot i \pitchfork 1 \cdot 1 \pitchfork(1 \pitchfork c)=e \cdot L \cdot i \pitchfork 1 \cdot 1 \pitchfork s t \cdot 1 \pitchfork(1 \pitchfork c) \\
& \stackrel{c C 4}{=} e \cdot 1 \pitchfork i \cdot 1 \pitchfork s t \cdot 1 \pitchfork(1 \pitchfork c) \stackrel{D}{=} e \cdot 1 \pitchfork i \cdot 1 \pitchfork e \\
& \stackrel{D}{=} 1 \bar{\pitchfork} \bar{l} \cdot e \cdot 1 \pitchfork e
\end{aligned}
$$

where 'VF2' means 'by Axiom VF2 for $T$ ' and 'CC4' means 'by Axiom CC4 for $\mathscr{V}$. Since $e \cdot 1$ the is monomorphic, this proves the desired commutativity.

Axiom CC5 says that the set mapping

$$
(A \bar{\pitchfork} A) \bar{V} \stackrel{(\bar{\imath}) \bar{V}}{\longrightarrow}(\bar{I} \bar{\pitchfork}(A \bar{\AA} A)) \bar{V}
$$

should send $1_{A}$ to $j_{A}$. We have

$$
\begin{aligned}
& i_{A \bar{A} A} \cdot e \cdot 1 \text { he } \stackrel{D}{=} i_{A \bar{T} A} \cdot \text { st } \cdot 1 \pitchfork\langle a, a\rangle \cdot 1 \text { he } \\
& \stackrel{D}{=} i_{A \bar{h} A} \cdot \mathrm{st} \cdot 1 \pitchfork e T \cdot 1 \pitchfork \lambda \cdot 1 \pitchfork(1 \pitchfork a) \\
& \stackrel{\text { st }}{=} i_{A \Phi_{A}} \cdot 1 \pitchfork e \cdot \mathrm{st} \cdot 1 \pitchfork \lambda \cdot 1 \pitchfork(1 \pitchfork a) \stackrel{i}{=} e \cdot i_{A \pitchfork_{A}} \cdot \mathrm{st} \cdot 1 \pitchfork \lambda \cdot 1 \pitchfork(1 \pitchfork a) \text {. }
\end{aligned}
$$

Applying $V$ to the right hand side of this equation gives a composite set mapping whose five constituents treat $1_{A}$ as follows 


$$
1_{A}-W \rightarrow 1_{A}-W \rightarrow j_{A}-W \rightarrow j_{A} T-W \rightarrow j_{A} T \cdot \lambda-W \rightarrow j_{A} T \cdot \lambda \cdot 1 \pitchfork a,
$$

using Axiom $\mathrm{CC} 5$ and $\mathrm{CC} 0$ for $\mathscr{V}$. By the definition (2.4), the right hand expression in (2.9) equals $j_{A} \cdot e$. So, by (2.8), $\left(\bar{i}_{A \bar{T}_{A}} \cdot e\right) V \cdot(1 \pitchfork e) V$ sends $1_{A}$ to $j_{A} \cdot e$. Since, by $\mathrm{CC} 0,(1 \hbar e) V$ is that injective mapping that multiplies on the right by $e$, we conclude that $\left(\bar{l}_{A} \bar{\phi}_{A} \cdot e\right) V$ sends $1_{A}$ to $j_{A}$. Since $e V$ is a chosen equalizer in the category of sets, i.e. an inclusion mapping, $\left(i_{A} \bar{A}_{A}\right) \widetilde{V}$ itself sends $1_{A}$ to $j_{A}$. This proves CC5 and thus the theorem.

\section{The closed adjointness of the functors}

Associated to the Eilenberg-Moore construction $\mathscr{V}_{\mathbf{0}}^{\mathbf{T}}$ there is a pair of adjoint functors $F \dashv U$ (with $F \cdot U=T$ ):

$$
\mathscr{V}_{0} \underset{U}{\stackrel{F}{\rightleftarrows}} \mathscr{V}_{0}^{\mathbf{T}}
$$

given on objects by $X F=\left(X T, \mu_{X}\right)$ and $(A, a) U=A(\mathscr{V}$ and $\mathbf{T}=T, \eta, \mu$ as in the preceding section). We shall show that $F$ and $U$ can be equipped with closedfunctor structure (with respect to the closed-category-structure given on $\mathscr{V}$ and the one constructed in the previous section on $\mathscr{V}^{\mathbf{T}}$ ). Then the front- and endadjunctions for the adjointness $F \dashv U$ will turn out to be closed transformations.

We first consider $F$. To make it a closed functor, we need a natural transformation

$$
\widehat{F}_{A B}:(A \pitchfork B) F \rightarrow A \overline{F \pitchfork} B F
$$

and a morphism

$$
F^{0}: \bar{I} \rightarrow I F \text {. }
$$

We define $\hat{F}_{A B}$ (strictly $\left.\hat{F}_{A B} U\right)$ by

$$
\widehat{F}_{A B} \cdot e=\widehat{T}_{A B},
$$

( $\hat{T}$ defined in $(2.2))$. To verify this factorization, we have to prove

We have

$$
\hat{T}_{A B} \cdot \mathrm{st} \cdot 1 \pitchfork \mu_{B}=\hat{T}_{A B} \cdot \mu_{A} \pitchfork 1 \text {. }
$$

$$
\begin{aligned}
& \hat{T} \cdot \mathrm{st} \cdot 1 \pitchfork \mu_{B} \stackrel{D}{=} \lambda \cdot \mathrm{st} \cdot 1 \pitchfork \mu \cdot \mathrm{st} \cdot 1 \pitchfork \mu \stackrel{\mathrm{st}}{=} \lambda \cdot \mathrm{st} \cdot \mathrm{st} \cdot 1 \pitchfork \mu T \cdot 1 \pitchfork \mu \\
& \stackrel{(2.1)}{=} \lambda \cdot \mathrm{st} \cdot \mathrm{st} \cdot 1 \pitchfork \mu \cdot 1 \pitchfork \mu \stackrel{*}{=} \lambda \cdot \mathrm{st} \cdot \mu \pitchfork 1 \cdot 1 \pitchfork \mu \\
& =\lambda \cdot \mathrm{st} \cdot 1 \pitchfork \mu \cdot \mu \pitchfork 1 \stackrel{D}{=} \hat{T} \cdot \mu \pitchfork 1,
\end{aligned}
$$

the equality sign marked $*$ by $\mathscr{V}$-naturality of $\mu$. This shows that (3.3) makes sense. We have to verify that $\hat{F}$ thus defined is a homomorphism. We have to prove

$$
\mu_{A \pitchfork_{B}} \cdot \hat{F}_{A B}=\left(\hat{F}_{A B}\right) T \cdot\left\langle\mu_{A}, \mu_{B}\right\rangle .
$$


We have

$$
\begin{aligned}
& \mu \cdot \hat{F} \cdot e \cdot \stackrel{D}{=} \mu \cdot \hat{T} \stackrel{D}{=} \mu \cdot(\mathrm{st}) T \cdot \lambda \cdot 1 \pitchfork \mu \stackrel{\mu}{=}(\mathrm{st}) T^{2} \cdot \mu \cdot \lambda \cdot 1 \pitchfork \mu \\
& \stackrel{1.6}{=}(\mathrm{st}) T^{2} \cdot \lambda T \cdot \lambda \cdot 1 \pitchfork \mu \cdot 1 \pitchfork \mu \stackrel{(2.1)}{=}(\mathrm{st}) T^{2} \cdot \lambda T \cdot \lambda \cdot 1 \pitchfork \mu T \cdot 1 \pitchfork \mu \\
& \stackrel{\lambda}{=}(\mathrm{st}) T^{2} \cdot \lambda T \cdot(1 \pitchfork \mu) T \cdot \lambda \cdot 1 \pitchfork \mu \stackrel{D}{=}(\widehat{T}) T \cdot \lambda \cdot 1 \pitchfork \mu \\
& \stackrel{D}{=}(\hat{F}) T \cdot e T \cdot \lambda \cdot 1 \pitchfork \mu \stackrel{D}{=}(\hat{F}) T \cdot\langle\mu, \mu\rangle \cdot e ;
\end{aligned}
$$

since $e$ is a monomorphism, this proves (3.4).

For $F^{0}$ in (3.2) we take $1_{I T}$ which clearly is a homomorphism.

Proposition 3.1. The data (3.1) and (3.2) makes $F$ into a closed functor.

Proof. We have to verify the axioms CF1-CF3 in [2]. Axiom CF1 says that the diagram

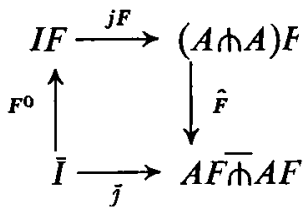

should commute, i.e. that $j T \cdot \hat{F}=j$. We have

$$
j \cdot e \stackrel{D}{=} j T \cdot \lambda \cdot 1 \pitchfork \mu \stackrel{V F 1}{=} j T \cdot(\mathrm{st}) T \cdot \lambda \cdot 1 \pitchfork \mu \stackrel{D}{=} j T \cdot \hat{T} \stackrel{D}{=} j T \cdot \hat{F} \cdot e
$$

the equality sign marked 'VF1' by Axiom VF1 for $T$. Since $e$ is a monomorphism, the result follows.

Axiom CF2 says that the diagram

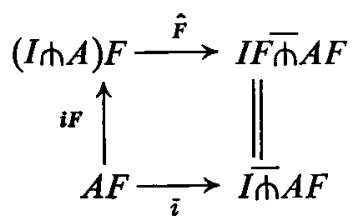

should commute. We have

$$
i T \cdot \hat{F} \cdot e \stackrel{D}{=} i T \cdot \hat{T} \stackrel{D}{=} i T \cdot \lambda \cdot \mathrm{st} \cdot 1 \pitchfork \mu \stackrel{1.7}{=} i \cdot \mathrm{st} \cdot 1 \mathrm{~h} \mu \stackrel{D}{=} i \cdot e .
$$

Since $i T=i F$ and $e$ is a monomorphism, CF2 follows.

Axiom CF3 says that the diagram

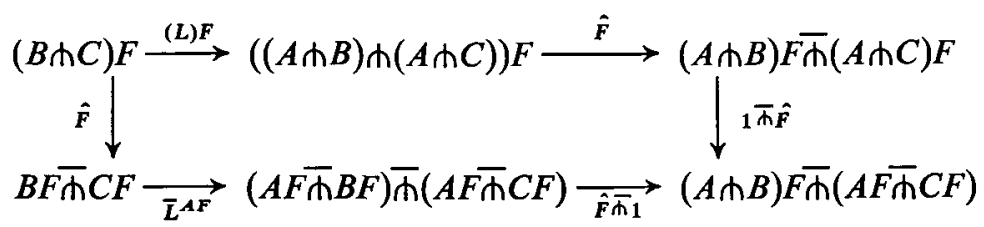

should commute. The essential step to prove this is the commutativity of the cor- 
responding CF3-diagram for $T$ (remove the upperbars in (3.5) and replace $F$ by $T$, $\hat{F}$ by $\hat{T})$. We state this formally as

Lemma 3.2. The $\hat{T}_{A B}$ defined in (2.2) satisfies Axiom CF3 for $T, \hat{T}$.

Proof. Using the lower composite in (2.2) to define $\hat{T}$, this amounts to proving

$$
\begin{aligned}
& L^{A} T \cdot \lambda \cdot \mathrm{st} \cdot 1 \pitchfork \mu \cdot 1 \pitchfork \lambda \cdot 1 \pitchfork \mathrm{st} \cdot 1 \pitchfork(1 \pitchfork \mu) \\
& =\lambda \cdot \mathrm{st} \cdot 1 \pitchfork \mu_{C} \cdot L^{A T} \cdot\left(1 \pitchfork \mu_{B}\right) \pitchfork 1 \cdot \operatorname{st} \pitchfork 1 \cdot \lambda \pitchfork 1 .
\end{aligned}
$$

We have

$$
\begin{aligned}
& L^{A} T \cdot \lambda \cdot \mathrm{st} \cdot 1 \pitchfork \mu \cdot 1 \pitchfork \lambda \cdot 1 \pitchfork \mathrm{st} \cdot 1 \pitchfork(1 \pitchfork \mu) \\
& \stackrel{1.6}{=} L^{A} T \cdot \lambda \cdot \mathrm{st} \cdot 1 \pitchfork \lambda T \cdot 1 \pitchfork \lambda \cdot 1 \pitchfork(1 \pitchfork \mu) \cdot 1 \pitchfork \mathrm{st} \cdot 1 \pitchfork(1 \pitchfork \mu) \\
& \stackrel{\text { st }}{=} L^{A} T \cdot \lambda \cdot 1 \pitchfork \lambda \cdot \mathrm{st} \cdot 1 \pitchfork \lambda \cdot 1 \pitchfork(1 \pitchfork \mu) \cdot 1 \pitchfork \mathrm{st} \cdot 1 \pitchfork(1 \pitchfork \mu) \\
& \stackrel{1.3}{=} \lambda \cdot L^{A} \cdot \mathrm{st} \cdot 1 \pitchfork \lambda \cdot 1 \pitchfork(1 \pitchfork \mu) \cdot 1 \pitchfork \mathrm{st} \cdot 1 \pitchfork(1 \pitchfork \mu) \\
& \stackrel{*}{=} \lambda \cdot \mathrm{st} \cdot L^{A} \cdot \lambda \pitchfork 1 \cdot 1 \pitchfork(1 \pitchfork \mu) \cdot 1 \pitchfork \mathrm{st} \cdot 1 \pitchfork(1 \pitchfork \mu) \\
& =\lambda \cdot \mathrm{st} \cdot L^{A} \cdot 1 \pitchfork(1 \pitchfork \mu) \cdot 1 \pitchfork \mathrm{st} \cdot \lambda \pitchfork 1 \cdot 1 \pitchfork(1 \pitchfork \mu) \\
& \stackrel{\underline{L}}{=} \lambda \cdot \mathrm{st} \cdot 1 \mathrm{\pitchfork} \mu \cdot L^{A} \cdot 1 \mathrm{hst} \cdot \lambda \pitchfork 1 \cdot 1 \mathrm{~h}(1 \pitchfork \mu) \\
& =\lambda \cdot \mathrm{st} \cdot 1 \pitchfork \mu \cdot L^{A} \cdot 1 \pitchfork \mathrm{st} \cdot 1 \pitchfork(1 \pitchfork \mu) \cdot \lambda \pitchfork 1 \\
& \stackrel{* *}{=} \lambda \cdot \mathrm{st} \cdot 1 \pitchfork \mu \cdot \mathrm{st} \cdot L^{A T} \cdot \mathrm{sth} 1 \cdot 1 \pitchfork(1 \pitchfork \mu) \cdot \lambda \pitchfork 1 \\
& =\lambda \cdot \mathrm{st} \cdot 1 \pitchfork \mu \cdot \mathrm{st} \cdot L^{A T} \cdot 1 \pitchfork(1 \pitchfork \mu) \cdot \operatorname{st} \pitchfork 1 \cdot \lambda \pitchfork 1 \\
& \stackrel{\text { st }}{=} \lambda \cdot \mathrm{st} \cdot \mathrm{st} \cdot 1 \pitchfork \mu T \cdot L^{A T} \cdot 1 \pitchfork(1 \pitchfork \mu) \cdot \mathrm{st} \pitchfork 1 \cdot \lambda \pitchfork 1 \\
& \stackrel{L}{=} \lambda \cdot \mathrm{st} \cdot \mathrm{st} \cdot 1 \mathrm{~h} \mu T \cdot 1 \mathrm{~h} \mu \cdot L^{A T} \cdot \mathrm{sth} 1 \cdot \lambda \pitchfork 1 \\
& \stackrel{(2.1)}{=} \lambda \cdot \mathrm{st} \cdot \mathrm{st} \cdot 1 \mathrm{~h} \mu \cdot 1 \pitchfork \mu \cdot L^{A T} \cdot \mathrm{sthl} \cdot \lambda \pitchfork 1 \\
& \stackrel{* * *}{=} \lambda \cdot \mathrm{st} \cdot \mu \pitchfork 1 \cdot 1 \pitchfork \mu \cdot L^{A T} \cdot \operatorname{sth1} \cdot \lambda \pitchfork 1 \\
& =\lambda \cdot \mathrm{st} \cdot 1 \pitchfork \mu \cdot \mu \pitchfork 1 \cdot L^{A T} \cdot \mathrm{st} \pitchfork 1 \cdot \lambda \pitchfork 1 \\
& \underline{\underline{L}} \lambda \cdot \mathrm{st} \cdot 1 \mathrm{~h} \mu \cdot L^{A T} \cdot(1 \mathrm{~h} \mu) \pitchfork 1 \cdot \operatorname{st} \pitchfork 1 \cdot \lambda \pitchfork 1 .
\end{aligned}
$$

The equality signs marked $* * *$, and $* * *$ by $\mathscr{V}$-naturality of $\lambda$, st, and of $\mu$, respectively. This proves the lemma. Note that commutativity of the monad was not used, in fact, $I \stackrel{\eta_{I}}{\rightarrow} I T$ and $\hat{T}=\lambda \cdot \mathrm{st} \cdot 1 \pitchfork \mu$ makes $T$ into a closed functor without assuming commutativity of the monad. We now turn to the proof of commutativity of (3.5). We have

$$
\begin{aligned}
& \hat{F} \cdot L^{A F} \cdot \hat{F} \bar{\pitchfork} 1 \cdot e \cdot 1 \pitchfork e \stackrel{D}{=} \hat{F} \cdot \bar{L} \cdot e \cdot \hat{F} \pitchfork 1 \cdot 1 \pitchfork e \\
& =\hat{F} \cdot \bar{L} \cdot e \cdot 1 \pitchfork e \cdot \hat{F} \pitchfork 1 \stackrel{D}{=} \hat{F} \cdot e \cdot L \cdot e \pitchfork 1 \cdot \hat{F} \pitchfork 1 \stackrel{D}{=} \hat{T} \cdot L^{A T} \cdot \hat{T} \pitchfork 1 \\
& \stackrel{3.2}{=}\left(L^{A}\right) T \cdot \hat{T} \cdot 1 \pitchfork \hat{T} \stackrel{D}{=}(L) F \cdot \hat{F} \cdot e \cdot 1 \pitchfork \hat{F} \cdot 1 \pitchfork e \stackrel{D}{=}(L) F \cdot \hat{F} \cdot 1 \pitchfork \hat{F} \cdot e \cdot 1 \text { he. }
\end{aligned}
$$


Since $(L) F=(L) T$ and $e \cdot 1$ the is a monomorphism, the result follows. This proves the proposition.

Next we make $U$ into a closed functor. We need a natural transformation

$$
U_{A B}:(A \bar{\pitchfork} B) U \rightarrow A U \pitchfork B U=A \pitchfork B ;
$$

we take simply $e$ for $A, B$. Also, we need a morphism

$$
U_{0}: I \rightarrow \bar{I} U=I T
$$

here we take $\eta_{I}$.

Proposition 3.3. These data make $U$ into a closed functor.

Proof. Axiom CF1 says that the diagram

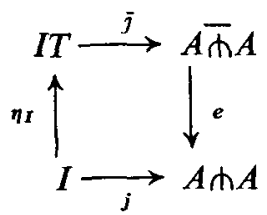

should commute. We have

$$
\eta \cdot j \cdot e \stackrel{D}{=} \eta \cdot j T \cdot \lambda \cdot 1 \pitchfork a \stackrel{\eta}{=} j \cdot \eta \cdot \lambda \cdot 1 \pitchfork a \stackrel{1.6}{=} j \cdot 1 \pitchfork \eta \cdot 1 \pitchfork a \stackrel{(2.3)}{=} j .
$$

Axiom CF2 says that the diagram

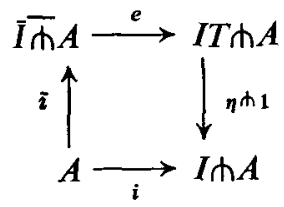

should commute. We have

$$
i \cdot e \cdot \eta \pitchfork 1 \stackrel{D}{=} i \cdot \mathrm{st} \cdot 1 \pitchfork a \cdot \eta \pitchfork 1=i \cdot \mathrm{st} \cdot \eta \pitchfork 1 \cdot 1 \pitchfork a \stackrel{*}{=} i \cdot 1 \pitchfork \eta \cdot 1 \pitchfork a \stackrel{(2.3)}{=} i,
$$

the equality sign marked $*$ by $\mathscr{V}$-naturality of $\eta$.

Finally, Axiom CF3 for $U$ says that the diagram (2.5) should commute, which it does by definition. This proves the proposition.

Let us consider the canonical front- and end-adjunctions for the adjoint pair $F, U$

$$
\begin{aligned}
& A \stackrel{\eta_{A}}{\longrightarrow} A F U=A T \\
& A U F \stackrel{\varepsilon_{A}=a}{\longrightarrow} A,
\end{aligned}
$$

where $A=(A, a)$.

Proposition 3.4. The transformations $\eta$ and $\varepsilon$ above are closed natural transformations (with respect to the above-mentioned closed structure of $F$ and $U$ ). 
Proof. We have to verify the axioms CN1 and CN2 of [2]. First, consider $\varepsilon$. Then $\mathrm{CN} 1$ says that the diagram

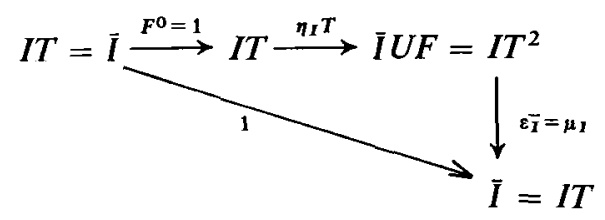

should commute (the top is the $\Phi^{0}$ associated to the functor $\Phi=U \cdot F$ according to Theorem I.3.1 of [2]). But this is just (2.1).

Axiom $\mathrm{CN} 2$ for $\varepsilon$ says that the diagram

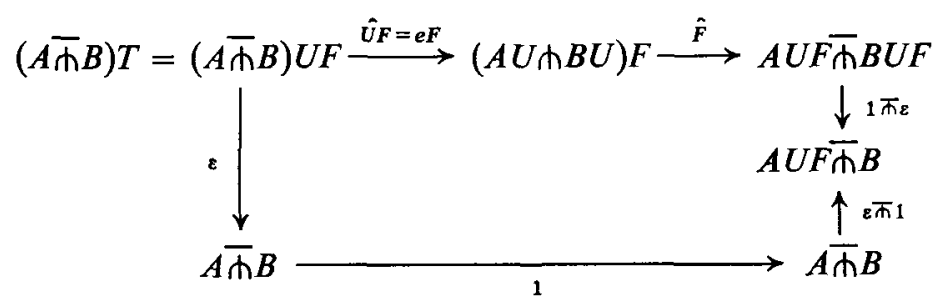

should commute. (The top is the $\hat{\phi}$ associated to the functor $\Phi=U \cdot F$, according to Theorem I.3.1 of [2].) It suffices to prove

We have

$$
\varepsilon \cdot \varepsilon \pitchfork 1 \cdot e=(e) F \cdot \hat{F} \cdot 1 \pitchfork \varepsilon \cdot e .
$$

$$
\begin{aligned}
& \varepsilon \cdot \varepsilon_{A} \bar{\pitchfork} 1 \cdot e \stackrel{D}{=} \varepsilon \cdot e \cdot \varepsilon_{A} \pitchfork 1 \stackrel{D}{=} \varepsilon \cdot e \cdot a \pitchfork 1 \\
& \stackrel{e}{=} \varepsilon_{A} \pitchfork B \cdot e \cdot \mathrm{st} \cdot 1 \pitchfork b \stackrel{D}{=}\langle a, b\rangle \cdot e \cdot \mathrm{st} \cdot 1 \pitchfork b \stackrel{D}{=} e T \cdot \lambda \cdot 1 \pitchfork b \cdot \mathrm{st} \cdot 1 \pitchfork b \\
& \stackrel{\mathrm{st}}{=} e T \cdot \lambda \cdot \mathrm{st} \cdot 1 \pitchfork b T \cdot 1 \pitchfork b \stackrel{(2.3)}{=} e T \cdot \lambda \cdot \mathrm{st} \cdot 1 \pitchfork \mu \cdot 1 \pitchfork b \\
& \stackrel{D}{=} e T \cdot \lambda \cdot \mathrm{st} \cdot 1 \pitchfork \mu \cdot 1 \pitchfork \varepsilon_{B} \stackrel{D}{=} e F \cdot \hat{T} \cdot 1 \pitchfork \varepsilon \stackrel{D}{=} e F \cdot \hat{F} \cdot e \cdot 1 \pitchfork \varepsilon \\
& \stackrel{D}{=} e F \cdot \hat{F} \cdot 1 \bar{\pitchfork} \varepsilon \cdot e .
\end{aligned}
$$

This proves $\mathrm{CN} 2$ for $\varepsilon$. The proof that $\eta$ is closed is easy: $\mathrm{CN} 1$ says here just $\eta_{I}=\eta_{I}$, which is true. Axiom CN2 says that the diagram

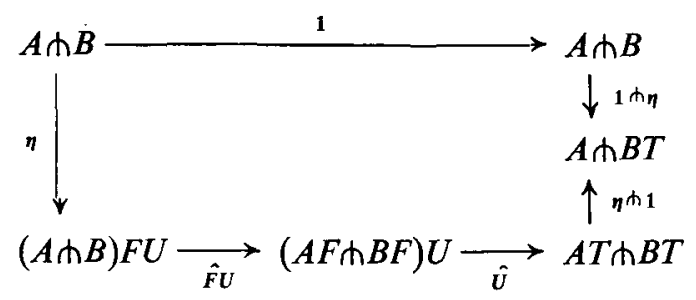

should commute. Here the bottom is $\hat{\Phi}$ associated to the composite functor $\Phi=F \cdot U$ according to Theorem I.3.1 of [2]. Since $\hat{U}=e, \hat{F} U \cdot \hat{O}=\hat{T}$, so we 
should prove that $\eta \cdot \hat{T} \cdot \eta \pitchfork 1=1 \pitchfork \eta$. We have

$$
\begin{aligned}
& \eta \cdot \hat{T} \cdot \eta \pitchfork 1 \stackrel{D}{=} \eta \cdot \lambda \cdot \mathrm{st} \cdot 1 \pitchfork \mu \cdot \eta \pitchfork 1=\eta \cdot \lambda \cdot \mathrm{st} \cdot \eta \pitchfork 1 \cdot 1 \pitchfork \mu \\
& \stackrel{*}{=} \eta \cdot \lambda \cdot 1 \pitchfork \eta \cdot 1 \pitchfork \mu \stackrel{(2.1)}{=} \eta \cdot \lambda \stackrel{1.6}{=} 1 \pitchfork \eta,
\end{aligned}
$$

the equality sign marked $*$ by $\mathscr{V}$-naturality of $\eta$. - This proves the proposition.

Corollary 3.5. A commutative monad $\mathbf{T}=(T, \eta, \mu)$ carries a canonical structure as closed monad:

$$
\begin{aligned}
& T^{0}=\eta_{I}: I \rightarrow I T \\
& \widehat{T}: \text { defined as before, }(2.2),
\end{aligned}
$$

(meaning that $T^{0}, \hat{T}$ makes $T$ into a closed functor in such a way that $\eta$ and $\mu$ become closed transformations).

PROof. According to Theorem 1.3.1 of [2] we can compose closed functors and transformations. In particular $T=F \cdot U$ has a composed closed structure which easily is seen to be the one given in the statement; and $\eta$ and $\mu=F \varepsilon U$ are closed transformations, since $\eta$ and $\varepsilon$ are, as we just proved.

We state without proof

Proposition 3.6. A monad is commutative in the sense defined here if and only if it is commutative in the sense of the previous paper [4]. In that case, the closedmonad structure given to $\mathbf{T}$ here is the same as the closed monad structure given in [4].

\section{References}

[1] S. Eilenberg and G. M. Kelly, 'A generalization of the functorial calculus', J. Algebra 3 (1966), 366-375.

[2] S. Eilenberg and G. M. Kelly, 'Closed Categories', Proc. of the Conference on Categorical Algebra, La Jolla, 1965 (Springer Verlag, 1966).

[3] S. Eilenberg and J. C. Moore, 'Adjoint functors and triples', Illinois J. Math. 9 (1965), 381398.

[4] A. Kock, 'Monads on symmetric monoidal closed categories', Arch. Math. 21 (1970), 1-10.

[5] J. Lambek, 'Deductive systems and categories', Math. Systems Theory 2 (1968), 287-318.

[6] F. E. J. Linton, 'Autonomous equational categories', J. Math. Mech. 15 (1966), 637-642.

The University of Aarhus

Denmark 